ISSN 0103-9954

\title{
DETERMINAÇÃO DA ROTA ÓTIMA DE TRANSPORTE COM AUXÍLIO DE UM SISTEMA DE INFORMAÇÃO GEOGRÁFICA
}

\author{
DETERMINATION OF THE BEST ROUTE OF TRANSPORT WITH AID OF A GEOGRAPHIC \\ INFORMATION SYSTEM
}

\author{
Paulo Costa de Oliveira Filho ${ }^{1}$ Eduardo da Silva Lopes ${ }^{2}$ Wagner Magraf ${ }^{3}$ Attilio Antonio Disperati ${ }^{4}$
}

\section{RESUMO}

O objetivo deste trabalho foi realizar uma análise comparativa entre os critérios de desempenho operacional do veículo e a menor distância na determinação da rota ótima no transporte florestal rodoviário, com auxílio de um sistema de informações geográficas. Inicialmente, foram realizadas a vetorização e a classificação de uma rede de estradas em uma empresa florestal. Em seguida, com auxílio do software Spring, versão 4.0, realizaram oito simulações de rota ótima de transporte, partindo-se de diferentes pontos locados ao acaso na área de estudo para um único destino. Os resultados indicaram que não houve diferença significativa na produtividade e no custo do transporte florestal entre os critérios analisados. Por outro lado, os resultados mostraram que o desempenho operacional foi o critério mais eficiente na determinação da rota ótima de transporte, comprovando a teoria de que nem sempre a menor distância é a mais econômica.

Palavras-chave: Transporte florestal; sistema de informação geográfica; rota ótima; custos de transporte.

\begin{abstract}
The objective of this work was to make a comparative analysis between the criteria of vehicle operational performance and lesser distance in the determination of the ideal route of forest road transportation, with aid of a geographic information system. Initially it was carried out the vector acquisition and the classification of a net of roads in a forest company. Subsequently, with aid of Spring software, version 4.0, there were conducted eight simulation of ideal route of transport, starting at different points romdomly located in the area of study and with a single destination. The results indicated that there is no significant difference of productivity and cost of transportation among the analyzed criteria. On the other hand, the results showed that the operational performance was the criteria most efficient in the determination of the ideal route of transport, proving the theory that not always the lesser distance is the most economic choice.
\end{abstract}

Key words: Forest transportation; geographic information system; ideal route; cost of transport.

\section{INTRODUÇÃO}

A malha rodoviária pública brasileira possui uma extensão aproximada de 1,89 milhões de quilômetros, na qual apenas $9 \%$ são pavimentados, e destes, $78 \%$ são classificados como deficiente ou ruim. Em média, $70 \%$ do transporte de cargas são realizados pelo modo rodoviário, enquanto nos países desenvolvidos a sua participação é de apenas 30\% (GEIPOT citado por Machado e Pereira, 2003). Segundo esses autores, as condições precárias da malha rodoviária brasileira contribuem para o aumento de $58 \%$ no consumo de combustível, $28 \%$ nos custos de manutenção, $100 \%$ no tempo de viagem e $50 \%$ nos acidentes de trânsito.

No setor florestal, o transporte de madeira é realizado quase que exclusivamente pelo modo rodoviário, por causa da extensa malha existente, oferta de veículos com diferentes capacidades de carga,

1. Engenheiro Florestal, Dr., Professor Adjunto do Departamento de Engenharia Florestal da Universidade Estadual do Centro-Oeste (UNICENTRO), Caixa Postal 21, CEP 84.500-000, Irati (PR). paulocostafh@irati.unicentro.br

2. Engenheiro Florestal, Dr., Professor Adjunto do Departamento de Engenharia Florestal da Universidade Estadual do Centro-Oeste (UNICENTRO), Caixa Postal 21, CEP 84.500-000, Irati (PR). eslopes@irati.unicentro.br

3. Engenheiro Florestal, Universidade Estadual do Centro-Oeste (UNICENTRO), Caixa Postal 21, CEP 84.500-000, Irati (PR). Bolsista de Iniciação Científica. margraf1981@bol.com.br

4. Engenheiro Florestal, PhD., Professor Adjunto do Departamento de Engenharia Florestal da Universidade Estadual do Centro-Oeste (UNICENTRO), Caixa Postal 21, CEP 84.500-000, Irati (PR).disperati@avalon.sul.com.br 
menor preço inicial, flexibilidade e probabilidade de escolha de rotas (Machado et al., 2000). Segundo Seixas (2001), o setor que vem sofrendo pressão de aumento nos custos em conseqüência da instalação de postos de pedágios, fiscalização rigorosa em relação à "Lei da Balança" e reajustes constantes nos preços dos combustíveis, representando, em média, de 40 a $70 \%$ do custo da madeira posta na fábrica.

Segundo Machado et al. (2000), os fatores que influenciam os custos de transporte florestal rodoviário são a distância, que determina o volume de madeira a ser transportado por turno de trabalho e o padrão de qualidade das estradas, que influencia o desempenho energético dos veículos de transporte, a durabilidade do veículo, a eficiência operacional, etc. O padrão das estradas florestais é o primeiro parâmetro a ser definido num projeto de construtivo visto que influencia os custos de construção, de manutenção e de transporte, especialmente por meio de suas geometrias horizontal e vertical, da qualidade da superfície da pista de rolamento e da largura.

Atualmente, o setor de estradas e transporte tem adquirido grande importância dentro do empreendimento florestal, uma vez que os custos do binômio estrada-transporte incidem significativamente sobre o valor final da madeira. Por isso, o uso de ferramentas como os Sistemas de Informação Geográfica (SIG's) são importantes na otimização do transporte florestal rodoviário. O uso dessa tecnologia de informação espacial associada às variáveis relacionadas às estradas (geometria horizontal e vertical, qualidade da superfície da pista, largura, etc), possibilita maior eficiência na tomada de decisão.

Bardal (1994) utilizou um SIG para elaborar projetos de estradas, reformulando os traçados existentes, de forma a otimizar o transporte de madeira contemplando variáveis técnicas e econômicas. Motta et al. (1996) demonstraram a viabilidade dos SIG's e da distância virtual na determinação da rota ótima de transporte florestal rodoviário. Nessa determinação, os autores utilizaram operadores de distância gerando diferentes cenários, constando-se diferenças entre rotas e veículos distintos no que concerne à otimização do transporte. Venturi (2000) utilizou o SIG para planejar a manutenção da malha rodoviária, enquanto Weber et al. (2003), para a avaliação de opções de traçado da estrada a ser pavimentada, considerando o uso do solo e altimetria do terreno como subsídios para simulações e tomada de decisão.

Este trabalho teve por objetivo fazer uma análise comparativa entre os critérios de desempenho operacional dos veículos e menor distância, visando à determinação da rota ótima de transporte florestal rodoviário com auxílio de um sistema de informação geográfica.

\section{MATERIAL E MÉTODOS}

\section{Área de estudo}

A área de estudo é localizada na Fazenda Nossa Senhora da Penha, pertencente à empresa Ripasa S/A Celulose e Papel, localizada no município de Lençóis Paulista, SP. A fazenda apresenta uma extensa malha viária, constituídas por estradas principais com revestimento primário e por estradas secundárias sem qualquer tipo de revestimento.

\section{Preparação dos dados gráficos}

Neste trabalho foi utilizado o software SPRING (Sistema de Processamento de Imagens Georreferenciadas) versão 4.0. De posse de um mosaico não-controlado de duas fotos aéreas métricas pancromáticas da fazenda na escala 1:25.000, procedeu-se à digitalização das estradas florestais e sua inserção no modelo de dados espacial do sistema de informações, como pode ser visto na Figura 1. 


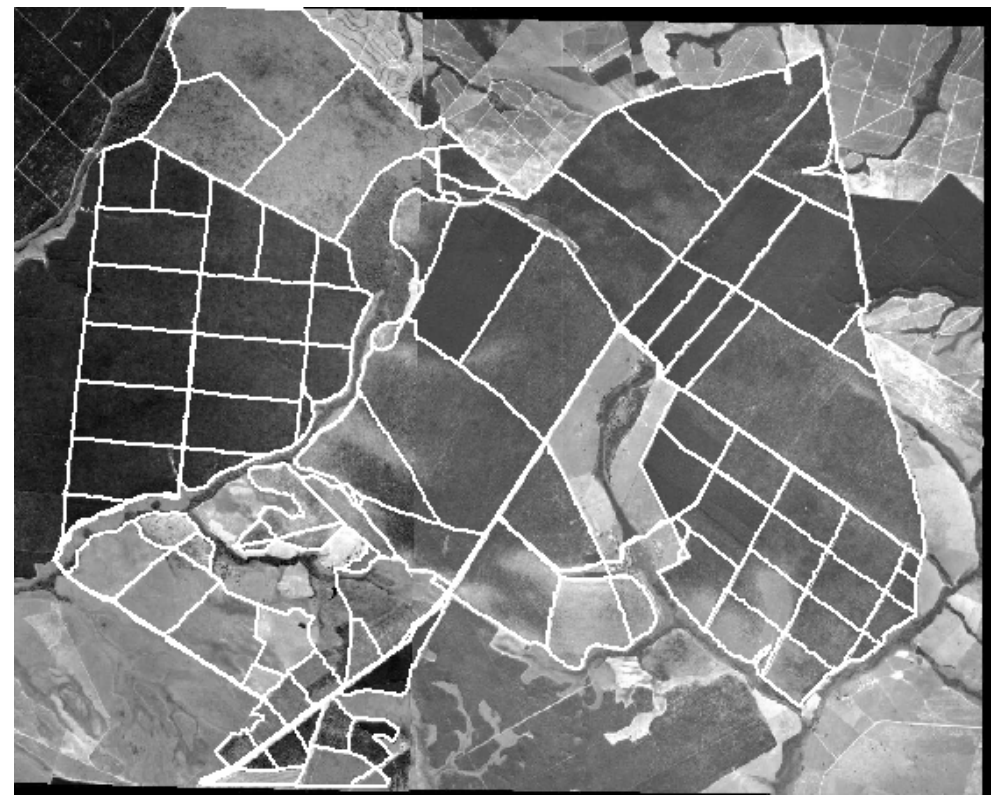

FIGURA 1: Mosaico fotográfico da área florestal e da malha viária utilizada no estudo. FIGURE 1: Photographic mosaic of the forest area and the road mesh used in the study.

Com base nas fotografias aéreas foi realizada a vetorização da rede de estradas, formando um plano de informação vetorial, classificado no modelo de dados como categoria do tipo rede. Em seguida, realizouse a preparação do arquivo vetorial representativo da rede pela da edição de linhas e a criação de nós ou ajustamento do modelo arco-nó, resultando em uma malha de arcos e nós interligados, de forma a permitir a realização de análises e simulações de roteamento.

\section{Classificação de estradas florestais}

De posse das fotografias aéreas com a rede de estradas vetorizada, realizou-se, de forma subjetiva, a estratificação das estradas da área de estudo em três categorias: Principal, Secundária e Terciária (Figura 2).

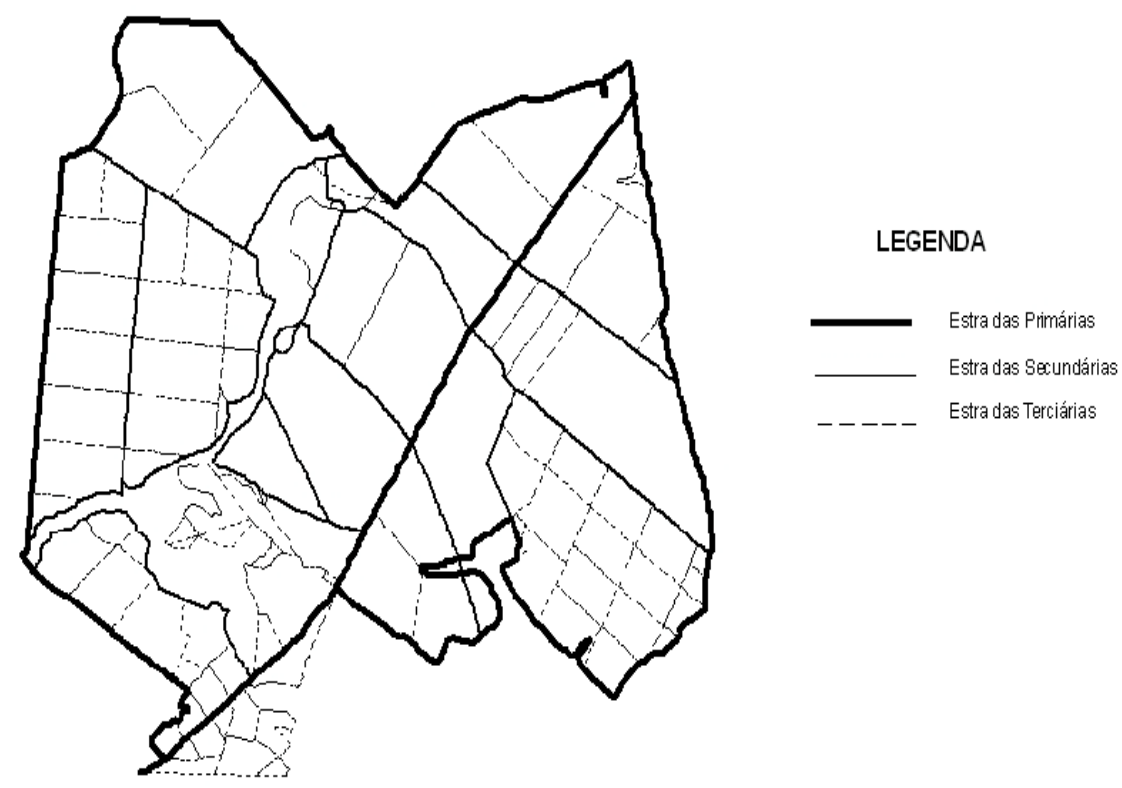

FIGURA 2: Malha viária evidenciando as categorias de estradas estabelecidas.

FIGURE 2: Road mesh evidencing the categories of established roads. 
A seguir, utilizando-se da classificação de estradas elaborada por Lopes et al. (2002), que estabeleceu vinte classes de estradas em razão da velocidade média de um veículo $6 \mathrm{x} \mathrm{4}$, com $200 \mathrm{cv}$ de potência e carregado com 20 toneladas, realizou-se o agrupamento em cada categoria estratificada, as classes possíveis de serem encontradas na área de estudo, conforme mostrado na Tabela 1.

TABELA 1: Categorias e classes de estradas florestais encontradas na área de estudo, com os respectivos parâmetros de qualidade e desempenho do veículo de transporte.

TABLE 1: Categories and class of forest roads found in the studied area, with the respective parameters of quality and performance of the transport vehicle.

\begin{tabular}{l|c|lll|}
\hline $\begin{array}{l}\text { Categoria } \\
\text { Estrada }\end{array}$ & Classe & \multicolumn{2}{|c|}{ Parâmetros de Qualidade } & $\begin{array}{c}\text { Velocidade } \\
\text { (km/hora) }\end{array}$ \\
\cline { 3 - 4 } Principal & 5 & Excelente & Pargura/Superficie Pista Rolamento & 41,0 \\
& 7 & Boa & Pista dupla revestimento primário & 38,6 \\
& 8 & Média & Pista dupla revestimento primário & 37,0 \\
& 10 & Ruim & Pista dupla revestimento primário & 35,6 \\
\hline \multirow{3}{*}{ Secundária } & 12 & Excelente & Pista dupla sem revestimento & 33,0 \\
& 14 & Boa & Pista dupla sem revestimento & 30,3 \\
& 16 & Média & Pista dupla sem revestimento & 28,7 \\
& 17 & Ruim & Pista dupla sem revestimento & 27,4 \\
\hline \multirow{3}{*}{ Terciária } & 15 & Excelente & Pista simples sem revestimento & 29,0 \\
& 18 & Boa & Pista simples sem revestimento & 26,6 \\
& 19 & Média & Pista simples sem revestimento & 24,3 \\
& 20 & Ruim & Pista simples sem revestimento & 20,9 \\
\hline
\end{tabular}

Fonte: Adaptado de Lopes et al. (2003)

\section{Estruturação do modelo de dados no SIG}

Após a edição do modelo arco-nó da rede e a posterior definição das categorias de estradas encontradas na área de estudo, elaborou-se um modelo de dados orientado ao objeto direcionado para a utilização das ferramentas de rede. Em seguida, realizou-se a associação dos arcos com os dados da Tabela 1, que foram estruturados no sistema como dados alfanuméricos não-espaciais do tipo atributo.

Cada objeto ou entidade arco-nó foi associado aos atributos referentes aos critérios classes de estradas inerentes ao desempenho operacional (velocidade) do veículo e à variável distância, associada ao comprimento das linhas. Tal procedimento foi realizado com o intuito de permitir a comparação e análise entre a rota ótima de transporte em razão do desempenho operacional do veículo (utilizando as classes de estrada) e menor distância de transporte (considerando-se o comprimento das linhas) que representam cada percurso.

\section{Determinação da rota ótima de transporte}

Foram realizadas oito simulações, partindo-se de diferentes pontos locados ao acaso na área de estudo, chegando-se em um único destino (indústria), de forma a obter a rota ótima de transporte pelos dos critérios:

a) Distância ótima de transporte associada ao desempenho operacional do veículo, considerando-se os seguintes parâmetros: geometria horizontal, largura da estrada, superfície da pista de rolamento e velocidade do veículo de transporte.

b) Distância ótima de transporte associada à menor distância entre o ponto de partida e chegada.

Em cada simulação dentro de cada critério analisado, determinaram-se a distância média do percurso, a velocidade média do veículo, o tempo médio de viagem no percurso e o tempo total do ciclo operacional. Esse último foi estimado em função do tempo de viagem carregado e vazio, obtido em função da velocidade média do veículo e pelas operações de carregamento e descarregamento, sendo este considerado em 45 minutos. 
Foram ainda estimados os custos do transporte florestal rodoviário em cada ciclo operacional, por quilômetro rodado, por tonelada transportada e por tonelada transportada por quilômetro. Para esses cálculos, considerou-se, com base na literatura especializada, um custo horário do veículo de transporte de R\$ $80,00 /$ hora para um veículo articulado.

\section{RESULTADOS E DISCUSSÃO}

A Figura 3 mostra a rota ótima de transporte obtida com base em três simulações realizadas para os critérios de desempenho operacional do veículo de transporte e menor distância.
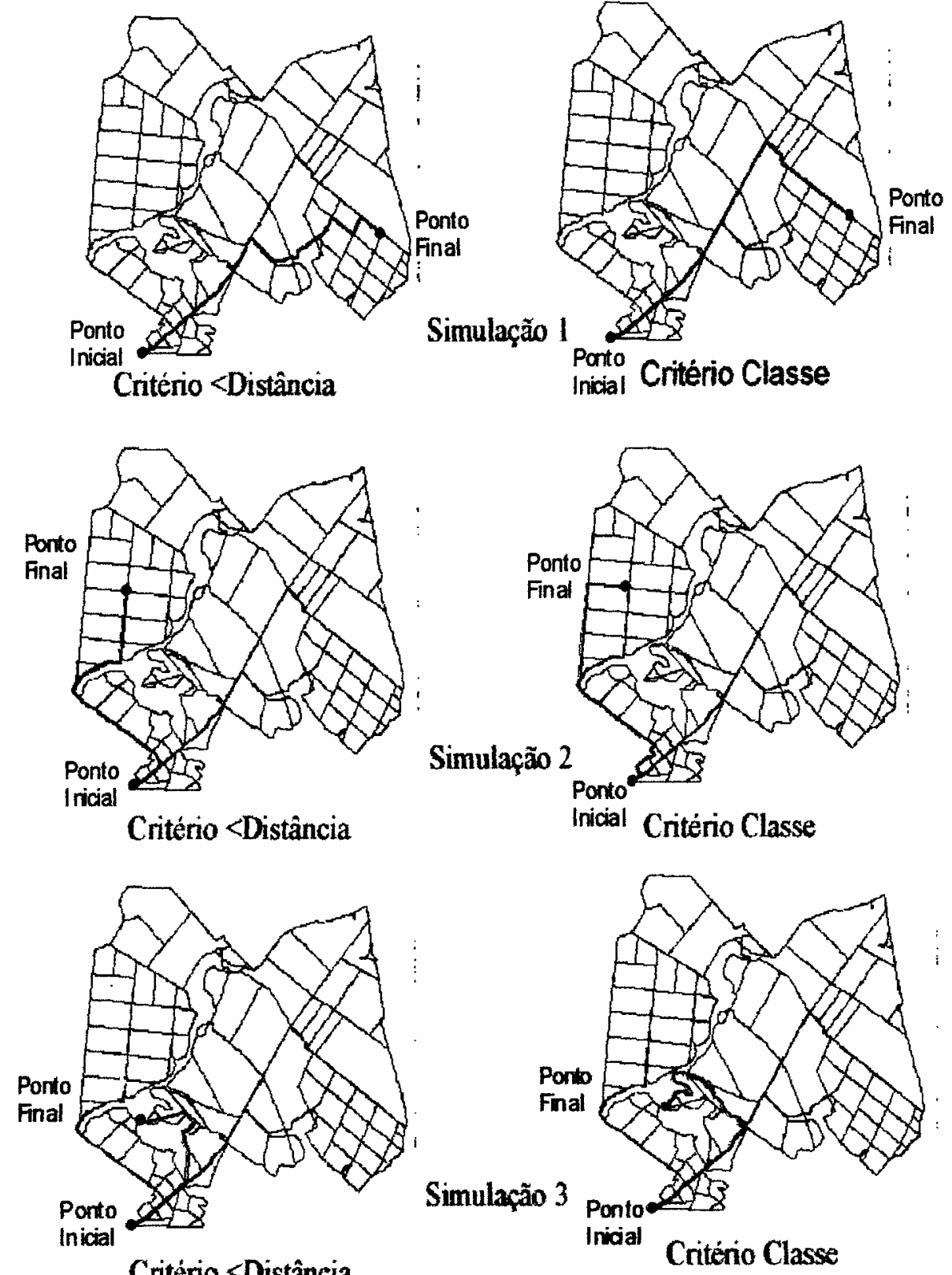

FIGURA 3: Simulação de transporte em diferentes rotas.

FIGURE 3: Simulation of transportation in different routes.

$\mathrm{Na}$ Tabela 2 são mostrados os resultados obtidos em relação à distância média do percurso, a velocidade média, o tempo médio de viagem e o tempo total do ciclo operacional do veículo de transporte nas simulações realizadas para os critérios de desempenho operacional e menor distância. 
TABELA 2: Resultados de oito simulações de percurso entre os talhões e a indústria.

TABLE 2: Results of eight simulation of passage ways between the forest stand and the industry.

\begin{tabular}{|c|c|c|c|c|c|c|c|c|c|c|}
\hline \multicolumn{3}{|c|}{ Simulação } & \multicolumn{8}{|c|}{ Critério Analisado } \\
\hline \multirow[b]{2}{*}{ Rota } & \multirow[b]{2}{*}{$\begin{array}{l}\text { Ponto } \\
\text { Início }\end{array}$} & \multirow[b]{2}{*}{$\begin{array}{c}\text { Ponto } \\
\text { Final }\end{array}$} & \multicolumn{4}{|c|}{ Desempenho Operacional } & \multicolumn{4}{|c|}{ Menor Distância } \\
\hline & & & $\begin{array}{l}\text { Distância } \\
(\mathrm{km})\end{array}$ & $\begin{array}{c}\text { Velocidade } \\
\text { Média } \\
(\mathrm{km} / \mathrm{h})\end{array}$ & $\begin{array}{l}\text { Tempo } \\
\text { Médio } \\
\text { viagem } \\
\text { (h) }\end{array}$ & $\begin{array}{c}\text { Tempo } \\
\text { Total } \\
\text { Ciclo } \\
\text { (h) }\end{array}$ & $\begin{array}{l}\text { Distância } \\
(\mathrm{km})\end{array}$ & $\begin{array}{l}\text { Velocidade } \\
\text { Média } \\
(\mathrm{Km} / \mathrm{h})\end{array}$ & $\begin{array}{l}\text { Tempo } \\
\text { Médio } \\
\text { viagem } \\
\text { (h) }\end{array}$ & $\begin{array}{c}\text { Tempo } \\
\text { Total } \\
\text { Ciclo } \\
\text { (h) }\end{array}$ \\
\hline 1 & 80 & 160 & 14,4 & 38,6 & 0,74 & 1,49 & 13,6 & 36,3 & 0,74 & 1,49 \\
\hline 2 & 31 & 160 & 11,8 & 37,7 & 0,62 & 1,37 & 11,1 & 35,8 & 0,62 & 1,37 \\
\hline 3 & 150 & 160 & 13,3 & 37,2 & 0,72 & 1,47 & 11,0 & 37,0 & 0,60 & 1,35 \\
\hline 4 & 152 & 160 & 17,2 & 37,3 & 0,92 & 1,67 & 13,5 & 35,2 & 0,76 & 1,51 \\
\hline 5 & 57 & 160 & 13,8 & 40,0 & 0,70 & 1,45 & 13,8 & 40,0 & 0,68 & 1,43 \\
\hline 6 & 14 & 160 & 14,1 & 34,9 & 0,82 & 1,57 & 13,8 & 39,7 & 0,70 & 1,45 \\
\hline 7 & 143 & 160 & 13,9 & 39,6 & 0,70 & 1,45 & 13,9 & 39,6 & 0,70 & 1,45 \\
\hline 8 & 38 & 160 & 16,5 & 37,7 & 0,88 & 1,63 & 15,1 & 34,6 & 0,88 & 1,63 \\
\hline Média & & & 14,4 & 37,9 & 0,76 & 1,51 & 13,2 & 37,3 & 0,72 & 1,46 \\
\hline Desvio & Padrão & & 1,7 & 1,6 & 0,10 & 010 & 1,43 & 2,18 & 0,09 & 0,09 \\
\hline
\end{tabular}

Como pode ser visto na Tabela 2, o critério de desempenho operacional na determinação da rota ótima de transporte permitiu ao veículo de transporte desenvolver uma maior velocidade média nos percursos $(37,9 \mathrm{~km} / \mathrm{h})$ em comparação ao critério da menor distância $(37,3 \mathrm{~km})$. No entanto, o tempo total médio de viagem por ciclo operacional foi superior $(1,51 \mathrm{~h})$ em relação ao critério da menor distância $(1,46 \mathrm{~h})$, por causa da maior distância média de percurso $(14,4 \mathrm{~km})$ nessas condições.

Apesar dos resultados obtidos entre os critérios não apresentarem diferenças significativas entre si pelo teste $\mathrm{t}(\mathrm{t}<0,05)$, a escolha do critério de desempenho operacional na definição da rota ótima de transporte poderá ser o critério mais eficaz. Isto em função de que, nessas condições, os veículos de transporte estarão operando em estradas de melhor qualidade, acarretando um menor desgaste dos veículos e redução dos custos de transporte, comprovados na Tabela 3.

TABELA 3: Resultados dos custos de transporte para as oito simulações realizadas.

TABLE 3: Results of transportation cost for the eight carried out simulations.

\begin{tabular}{|c|c|c|c|c|c|c|c|c|c|c|}
\hline \multicolumn{3}{|c|}{ Simulação } & \multicolumn{8}{|c|}{ Critério Analisado } \\
\hline \multirow[b]{2}{*}{ Rota } & \multirow[b]{2}{*}{$\begin{array}{l}\text { Ponto } \\
\text { Inicio }\end{array}$} & \multirow[b]{2}{*}{$\begin{array}{r}\text { Ponto } \\
\text { Final }\end{array}$} & \multicolumn{4}{|c|}{ Desempenho do Veículo } & \multicolumn{4}{|c|}{ Menor Distância } \\
\hline & & & $\begin{array}{c}\text { Custo } \\
\text { (R\$/ciclo) }\end{array}$ & $\begin{array}{c}\text { Custo } \\
(\mathrm{R} \$ / \mathrm{km})\end{array}$ & $\begin{array}{l}\text { Custo } \\
(\mathrm{R} \$ / \mathrm{t})\end{array}$ & $\begin{array}{c}\text { Custo } \\
(\mathrm{R} \$ / \mathrm{t} / \mathrm{km})\end{array}$ & $\begin{array}{c}\text { Custo } \\
\text { (R\$/ciclo) }\end{array}$ & $\begin{array}{c}\text { Custo } \\
(\mathrm{R} \$ / \mathrm{km})\end{array}$ & $\begin{array}{l}\text { Custo } \\
(\mathrm{R} \$ / \mathrm{t})\end{array}$ & $\begin{array}{c}\text { Custo } \\
(\mathrm{R} \$ / \mathrm{t} / \mathrm{km})\end{array}$ \\
\hline 1 & 80 & 160 & 119,20 & 4,14 & 5,96 & 0,21 & 119,20 & 4,38 & 5,96 & 0,22 \\
\hline 2 & 31 & 160 & 109,60 & 4,64 & 5,48 & 0,23 & 109,60 & 4,94 & 5,78 & 0,25 \\
\hline 3 & 150 & 160 & 117,60 & 4,42 & 5,88 & 0,22 & 108,00 & 4,91 & 5,40 & 0,25 \\
\hline 4 & 152 & 160 & 133,60 & 3,88 & 6,68 & 0,19 & 120,80 & 4,47 & 6,04 & 0,22 \\
\hline 5 & 57 & 160 & 116,00 & 4,20 & 5,80 & 0,21 & 114,40 & 4,14 & 5,72 & 0,21 \\
\hline 6 & 14 & 160 & 125,60 & 4,45 & 6,28 & 0,22 & 116,00 & 4,20 & 5,80 & 0,21 \\
\hline 7 & 143 & 160 & 116,00 & 4,17 & 5,80 & 0,21 & 116,00 & 4,17 & 5,80 & 0,21 \\
\hline 8 & 38 & 160 & 130,40 & 3,95 & 6,52 & 0,20 & 130,40 & 4,32 & 6,52 & 0,22 \\
\hline Média & & & 121,00 & 4,23 & 6,05 & 0,21 & 116,80 & 4,44 & 5,88 & 0,22 \\
\hline Desvi & Padrão & & 8,13 & 0,26 & 0,41 & 0,01 & 7,00 & 0,32 & 0,32 & 0,02 \\
\hline
\end{tabular}

Como pode ser observado, apesar do maior custo médio por tonelada transportada (R\$ 6,05/t), o custo médio por quilômetro foi ligeiramente inferior $(\mathrm{R} \$ 0,21 / \mathrm{t} / \mathrm{km})$ no critério do desempenho operacional. Apesar de não haver diferença significativa entre os critérios em relação aos custos de transporte teste $\mathrm{t}(\mathrm{t}<$ 0,05), a escolha da rota de transporte em função do critério de desempenho operacional, permitirá ao veículo, sobretudo em longas distâncias, obter ganhos significativos em termos de redução de custos. Tal fato pode ser explicado, pois nessas condições, o veículo estará operando em estradas de melhor qualidade, desenvolvendo uma velocidade média homogênea ao longo do percurso, acarretando inicialmente, na redução da necessidade de mudanças constantes de marcha, mantendo a rotação do motor mais homogênea, e 
conseqüentemente, reduzindo o consumo de combustível, desgastes excessivos e quebras de peças e componentes como freios, embreagens, pneus e transmissão.

Segundo Silveira (2003), o motor de um veículo de transporte ao trafegar em estradas de melhor qualidade, operando com rotações constantes, terá mais força disponível. Além disso, segundo estudos realizados pela Volvo, o consumo de combustível poderá ser reduzido em até $25 \%$, pela simples condução do veículo de forma mais suave, que poderá ser obtido em rotas que permitam ao veículo de transporte ter um melhor desempenho operacional, com velocidades mais constantes.

\section{CONCLUSÕES}

Com base nos resultados obtidos nesta pesquisa, as seguintes conclusões podem ser apresentadas:

a) O software SPRING revelou ser uma ferramenta com grande potencial no auxílio à tomada de decisão no transporte florestal rodoviário, sobretudo na determinação da rota ótima de transporte.

b) O modelo de dados orientado ao objeto desenvolvido para utilizar as ferramentas de rede, aplicadas ao transporte rodoviário florestal mostrou-se totalmente adequado e bastante funcional, especialmente se utilizarmos um software de domínio público que dispensa compromisso com maiores investimentos.

c) Apesar de que não houve diferença significativa entre os critérios estudados, os resultados obtidos mostraram que o desempenho operacional poderá ser o critério mais eficiente na determinação da rota ótima de transporte, pois nessa situação, o veículo poderá operar em estradas de melhor padrão de qualidade, acarretando um menor desgaste do conjunto e proporcionando redução dos custos de transporte, sobretudo em situações de transporte em larga escala.

d) Os resultados obtidos neste trabalho mostraram a aplicabilidade do critério de desempenho operacional na determinação da rota ótima de transporte, comprovando a teoria de que nem sempre a menor distância é a mais econômica na determinação da rota ótima de transporte florestal rodoviário.

\section{REFERÊNCIAS BIBLIOGRÁFICAS}

BARDDAL, S. M. A Utilização do SIG na Klabin. In: SEMINÁRIO DE ATUALIZAÇÃO EM SENSORIAMENTO REMOTO E SISTEMAS DE INFORMAÇÕES GEOGRÁFICAS APLICADOS À ENGENHARIA FLORESTAL, 1. , Curitiba, 1994. Anais... Curitiba, 1994. p. 83-92.

LOPES, E. S.; MACHADO, C. C.; SOUZA, A. P. Classificação e custos de estradas em florestas plantadas na região Sudeste do Brasil. Revista Árvore, Viçosa-MG, v. 26, n. 3, p. 329-338, 2002.

MACHADO, C. C.; LOPES, E. S.; BIRRO, M. H. Elementos básicos do transporte florestal rodoviário. Viçosa: UFV, 2000. 167p. il.

MACHADO, C. C.; PEREIRA, R. S. Qualidade da rodovia versus desempenho e custo do transporte rodoviário de madeira. In: SIMPÓSIO BRASILEIRO SOBRE COLHEITA E TRANSPORTE FLORESTAL, 6., 2003, Belo Horizonte. Anais... Belo Horizonte, MG: UFV/SIF, 2003. p. 132-149.

MOTTA, L. P. et. al. Utilização do sistema de informações geográficas e da distância virtual na otimização do transporte florestal rodoviário. Revista Árvore, Viçosa, v. 20, n. 3, p. 381-394, 1996.

SEIXAS, F. Novas tecnologias no transporte rodoviário de madeira. In: SIMPÓSIO BRASILEIRO SOBRE COlHeitA E TRANSPORTE FlORESTAL, 5. 2001, Porto Seguro, Anais... Porto Seguro, BA: UFV/SIF, 2001. p. $1-27$

SILVEIRA, G. L. Monitoramento do consumo de combustível de veículos de transporte rodoviário de madeira utilizando computador de bordo. 2003. 55p.. Dissertação (Mestrado em Ciência Florestal) - Universidade Federal de Viçosa, Viçosa, 2003

WEBER, E. et al Análise de alternativas de traçado de uma estrada utilizando rotinas de apoio à decisão em SIG. Disponível em : <http://www.delmonio.ecologia.ufrgs.br/idrisi/artigos/estrada.pdf. $>$ Acesso em: 22 set. 2003. 